\title{
Clonninger's temperament and character inventory profiles of anatomists: is there a relation with specialty choice?
}

\author{
Kosif $\mathrm{R}^{1}$, Konuk $\mathrm{N}^{2}$ \\ Abant Izzet Baysal University, Faculty of Medicine, Department of Anatomy, Bolu, Turkey. \\ rengink@yahoo.com
}

\begin{abstract}
Career psychologists have argued that the career choice and personality interfere with each other. There have been lots of investigations aimed at seeking the relationships between career interests and personality characteristics. There is limited knowledge on personality profiles of the anatomists and on how they are related with their specialty choices. AIM: In this research we aimed to explore the relationship between personality and career interests of anatomists. METHOD: Out of 279 anatomists who had been asked to complete the survey via e-mail including three questionnaires, 79 (53 male, 26 female) responded in the present study. Personality was assessed using the Cloninger's Temperament and Character Inventory $(\mathrm{TCl})$. The career interest was determined by Holland's Theme Codes. RESULTS: The order of high frequency Holland's Codes was as follows: social (44.3\%), realistic (35.4\%), investigative (27.8\%), conventional (19.0\%), artistic (7.6\%), and enterprising (5.1\%). With regard to temperament components of TCl was as follows: novelty seeking (mean $=17.7 \pm 4.7)$, harm avoidance (mean $=13.9 \pm 6.1$ ), reward dependence (mean $=13.2 \pm 3.4)$, and persistence $($ mean $=5.4 \pm 2.1)$. Character profiles are as follows: self-directedness ( mean $=33.1 \pm 6.3$ ), self-transcendence (mean $=17.9 \pm 7.6$ ), and cooperativeness (mean=30.6 \pm 5.9 ). According to the last questionnaire, the most important cause for choosing anatomy is the interest in anatomy since medical study time. CONCLUSION: These results in part support Holland's theory, which takes the career as a function of personality and the personality profiles of anatomists have affected the motivation to select their specialty choice partially (Tab. 3, Fig. 1, Ref. 10). Text in PDF www.elis.sk.

KEY WORDS: anatomists, personality, specialty choice.
\end{abstract}

\section{Introduction}

Personality has been reported to be related to medical career choice. The idea that distinct personality types may exist for physicians in different specialty areas has been examined using different personality inventories (1). Following graduation, physicians enter a variety of medical specialties that differ in work settings, job duties, requisite skills, and vocational interests. In fact, these specialties differ so much that they almost constitute distinct occupations (2).

Personality measures would not be particularly effective in predicting medical specialty choice. It also suggests that medical specialty selection should concentrate on task performance by measuring skills, self-efficacy, attitudes, and aptitudes rather than personality (3).

For every personality type, it is possible to find a satisfying match with more than one area of medicine. Medical specialties have been investigated using different personality inventories.

${ }^{1}$ Abant Izzet Baysal University, Faculty of Medicine, Department of Anatomy, Bolu, Turkey, and ${ }^{2}$ Istanbul University, Cerrahpaşa Medical Faculty, Department of Psychiatry, Istanbul, Turkey

Address for correspondence: Dr. R. Kosif, Department of Anatomy, Faculty of Medicine, Abant Izzet Baysal University, Gölköy Campus 14280, Bolu - Turkey.

Phone: +374.25346563043, Fax: +374.2534559

Acknowledgements: This study has been presented in 2014 IFAA Congress in Beijing at 8-10 Aug, as a poster.
If you are a visually oriented person, consider specialties like pathology, dermatology, and radiology. For students who want to speak only the language of medicine every day as a doctor's doctor, radiology and pathology are ideal choices (4).

Primary care specialties, like internal medicine and family practice, are great opportunities to have long-term, intimate patient relationships. If you prefer an action-oriented specialty that gives immediate gratification, then consider anesthesiology, any surgical subspecialty, and emergency medicine. Some areas overlap considerably, like the great variety of medical problems encountered in both family practice and emergency medicine. But at the same time, they can have significant differences, like the long-term follow-up nature of family practice versus the acute, stabilize-thepatient-and-move-on style of emergency medicine (4).

Physicians represent a homogenous group in terms of intellectual and cognitive ability and seem to share common personality traits based on their choice of medicine as a career $(2,5)$. Anesthesiologists, family practitioners, obstetricians and gynecologists, pediatricians, physiatrists, psychiatrists, surgeons and internists have been investigated as to their personality types (1).

There has been much investigation aimed at seeking the relationships between career interests and personality characteristics. There is no knowledge on personality profiles of anatomists and on how they are related to their specialty choices. 


\section{Material and method}

In this research we aimed to explore the relationship between personality and career interest of anatomists. Questionnaire forms were sent to anatomists after receiving the approval from AİBÜ Social Sciences Human Research Ethic Committee (Protocol No: 2013/56).

Out of 279 anatomists who had been asked to complete the survey via e-mail including three questionnaires. 79 (53 male, 26 female; $67.1 \%$ men and $32.9 \%$ women) responded in the present study.

\section{First questionnaire}

Personality was assessed using the Cloninger's Temperament and Character Inventory (TCI; a 240-question, self-administered, true-false questionnaire). TCI test (TCI test and TCI rating scale) in Turkish version is used for the questionnaire $(6,7)$.

\section{TCI Scores}

There are 240 statements that vary depending on own interests, emotional reactions, attitudes, aims, and values. The analysis of the questionnaire allows the calculation of the main scores corresponding to four temperament dimensions and three character dimensions as follows: novelty seeking (NS), harm avoidance (HA), reward dependence (RD), persistence (P), self-directedness (SD), cooperativeness (C), self-transcendence (ST).

\section{Looking at the big picture}

Novelty seeking (NS)

Exploratory excitability (NS1), impulsiveness (NS2), extravagance (NS3), disorderliness (NS4);

Harm avoidance (HA)

Anticipatory worry (HA1), fear of uncertainty (HA2), shyness (HA3), fatigability (HA4)

Reward dependence (RD)

Sentimentality (RD1), openness to warm communication (RD2), attachment (RD3), dependence (RD4)

\section{Persistence $(P)$}

Eagerness of effort (PS1), work-hardened (PS2), ambitious (PS3), perfectionist (PS4)

Self-directedness (SD)

Responsibility (SD1), purposeful (SD2), resourcefulness (SD3), self-acceptance (SD4), enlightened second nature (SD5) Cooperativeness $(C)$

Social acceptance (C1), empathy (C2), helpfulness (C3), compassion (C4), pure-hearted conscience (C5) Self-transcendence (ST)

Self-forgetfulness (ST1), transpersonal identification (ST2), spiritual acceptance (ST3).

Cloninger et al identified four temperament dimensions as follows: novelty seeking (NS), harm avoidance (HA), reward dependence (RD), and persistence $(\mathrm{P})$, which are genetically independent of one another. HA is the tendency to inhibit activity when faced with punishment, novelty, or no reward. Persons with high HA fear new situations and become inhibited. They are shy with new people. If punished or not rewarded, they stop the punished or non-rewarded behavior and try to "blend into the woodwork". NS is the tendency to explore, to be curious about novel stimuli, and to actively avoid the punishment and frustrating non-reward. High novelty seekers are curious about new situations, and the riskier the situation, the greater the "buzz" they experience. If punished, they leave or become aggressive. If not rewarded, they become bored and leave. RD is the tendency to develop behaviors that lead to positive reinforcement and to maintain rewarded behaviors. Persons with high RD want to please others and social institutions that were previously rewarding, and tend to conform to the reward system. $\mathrm{P}$ is the tendency to maintain behaviors.

Cloninger et al's model also includes three character dimensions: self-directedness (SD), cooperativeness (C), and self-transcendence (ST). In contrast to temperament which involves differences in autonomic systems, the characterological aspects of personality involve individual differences in goals and values. SD is the tendency to perceive reinforcement as contingent on one's own actions, and to adapt one's behavior according to one's goals; that is, to be autonomous. Persons high on SD are purposeful and resourceful. Persons low on SD are unreliable, ineffective, and dependent. $\mathrm{C}$ is the tendency to perceive reinforcement as contingent on others' responses and to be able to identify with others and accept them; that is, to be "part of a group." Persons high on $\mathrm{C}$ are empathic, helpful, and compassionate. Persons low on $\mathrm{C}$ is intolerant and vengeful. Persons very high on $\mathrm{C}$ may "live for others". Persons very low on C may be antisocial. ST is the tendency to perceive reinforcement as contingent on forces (e.g., God, nature, biology, or fate) outside of one's control, and a tendency to have a belief system and to feel part of nature and the universe. Persons high on ST are philosophical and may be unconventional. Persons low on ST is often unimaginative and conventional.

\section{Second questionnaire}

The Holland Codes or the Holland Occupational Themes (RIASEC) is a theory of careers and vocational choice based upon 5 personality types as follows: realistic (doers), investigative (thinkers), artistic (creators), social (helpers), enterprising (persuaders), and conventional (organizers) (8).

Doers (realistic) are independent, stable, persistent, genuine, practical, and thrifty in tasks that are tactile, physical, athletic, or mechanical, being outdoors, using tools, operating machines, interacting with animals, and working with their hands.

Thinkers (investigative) are intellectual, introspective, and inquisitive. They are curious, methodical, rational, analytical, and logical, scholarly, scientific, technical, or medical avid readers. They like to solve problems, perform experiments, and conduct research.

Creators (artistic) are creative, intuitive, sensitive, articulate, and expressive. They are unstructured, original, nonconforming, and innovative. They rely on feelings, imagination, and inspiration. They like to work with ideas, abstractions, and concepts. They are spontaneous and open-minded.

Helpers (social) are kind, generous, cooperative, patient, caring, helpful, empathetic, tactful, and friendly. They excel in socializing, helping others, and teaching. They like tasks that involve teamwork, social interaction, relationship building, and improvement of society. 
Tab. 1. Descriptive statistics for the TCI facet scales.

\begin{tabular}{|c|c|c|c|c|c|}
\hline & $\mathrm{n}$ & Mean & SD & Min. & Max. \\
\hline Novelty seeking & 79 & 17.785 & 4.779 & 8 & 29 \\
\hline Exploratory excitability & 79 & 6.266 & 2.182 & 2 & 11 \\
\hline Impulsiveness & 79 & 3.671 & 1.872 & 0 & 9 \\
\hline Extravagance & 79 & 4.456 & 1.686 & 1 & 8 \\
\hline Disorderliness & 79 & 3.392 & 1.605 & 0 & 7 \\
\hline Harm avoidance & 79 & 13.911 & 6.125 & 1 & 25 \\
\hline Anticipatory worry & 79 & 4.722 & 1.761 & 0 & 9 \\
\hline Fear of uncertainty & 79 & 3.367 & 2.045 & 0 & 7 \\
\hline Shyness & 79 & 3.215 & 2.319 & 0 & 8 \\
\hline Fatigability & 79 & 2.608 & 2.097 & 0 & 7 \\
\hline Reward dependence & 79 & 13.215 & 3.452 & 6 & 20 \\
\hline Sentimentality & 79 & 6.620 & 1.957 & 2 & 10 \\
\hline Attachment & 79 & 4.101 & 1.802 & 1 & 7 \\
\hline Persistence & 79 & 5.430 & 2.188 & 1 & 8 \\
\hline Self directedness & 79 & 33.152 & 6.395 & 14 & 44 \\
\hline Responsibility - Condemnation & 79 & 5.924 & 1.678 & 2 & 8 \\
\hline Purposefulness & 79 & 6.051 & 1.592 & 2 & 8 \\
\hline Resourcefulness & 79 & 4.101 & 1.081 & 1 & 5 \\
\hline Self acceptance - Own contention & 79 & 7.380 & 2.554 & 1 & 11 \\
\hline Enlightened second nature & 79 & 9.696 & 2.102 & 1 & 12 \\
\hline Cooperativeness & 79 & 30.658 & 5.993 & 16 & 42 \\
\hline Social Acceptance - Social Intolerance & 79 & 6.329 & 1.591 & 3 & 8 \\
\hline Empathy - Social indifference & 79 & 4.873 & 1.314 & 2 & 7 \\
\hline Helpfulness & 79 & 5.241 & 1.361 & 2 & 8 \\
\hline Compassion - Revenge & 79 & 6.772 & 2.567 & 1 & 10 \\
\hline Pure-hearted conscience - Self benefit & 79 & 7.443 & 1.206 & 5 & 9 \\
\hline Transpersonal identification - Self decomposition & 79 & 5.114 & 1.860 & 2 & 9 \\
\hline Spirituel acceptance - Rational materialism & 79 & 7.000 & 3.475 & 1 & 13 \\
\hline
\end{tabular}

Persuaders (enterprising) are adventurous, ambitious, assertive, extroverted, energetic, enthusiastic, confident, and optimistic. They are dominant, persuasive, and motivational. They like influencing others, being in charge, taking risks, debating and competing.

\section{Third questionnaire \\ Reasons for choosing anatomy (Supp 1)}

\section{Statistical analysis}

In order to evaluate the findings in the study, SPSS statistics package program was used for the statistical analysis. Besides the descriptive statistical methods (frequency, percentage, average, standard deviation), Kolmogorov-Smirnov test was used in order to analyze the regular range during the evaluation of the data of the study. In the case that there were two groups in the comparison of quantitative data, Mann-Whitney U test was used in the intergroup comparison of parameters which did not show a regular range. In the case that there were two groups in the comparison of quantitative data, Kruskal-Wallis test was used and Mann-Whitney U test was used in order to determine the group which caused the difference. The results were evaluated in the confidence interval of $95 \%$ and the significance level of $\mathrm{p}<0.05$.

\section{Results}

Twenty-six (32.9\%) of the anatomists who answered the questionnaire were female, while $53(67.1 \%)$ of them were male. Twenty-two $(27.8 \%)$ of them were $<35 ; 9(11.4 \%)$ were $36-40$; $11(13.9 \%)$ were $41-45 ; 23(29.1 \%)$ were $46-50 ; 14(17.7 \%)$ were $>51$ years old. Thirty-five $(44.3 \%)$ of the anatomists had a TUS degree (medical license examination in Turkey), 36 (45.6 $\%)$ had doctorate, $8(10.1 \%)$ had master + doctorate degrees. 3 (3.8 \%) were TUS assistants (Research Assistant), 4 (5.1\%) were doctorate students, 1 (1.3\%) was an instructor, 2 (2.5\%) were specialists, $15(19.0 \%)$ were assistant professors, 18 (22.8 $\%)$ were associate professors, $36(45.6 \%)$ were professors, 60 (75.9\%) were graduated from Medical Faculty, and 19 (24.1\%) graduated from other faculties.

\section{TCI scores}

Table 1 - descriptive statistics for TCI facet scales

With regard to temperament components of TCI, the mean values were as follows: novelty seeking (mean=17.7 \pm 4.7 ), harm avoidance (mean=13.9 \pm 6.1 ), reward dependence (mean=13.2 \pm 3.4 ), and persistence $($ mean $=5.4 \pm 2.1)$. Character profiles were as fol- 
lows: self-directedness (mean $=33.1 \pm 6.3$ ), cooperativeness (mean=30.6 \pm 5.9 ), self-transcendence (mean=17.9 \pm 5.6 ).

Differences according to age range $(<35,36-40,41-45$, 46-50, > 51):

The scores of fatigability ( $4.21 \pm 2.32)$ of anatomists aged $>51$ are higher than those of anatomists aged $<50$. The scores of sentimentality $(8.11 \pm 0.78)$ of anatomists aged $36-40$ are higher than those of anatomists in other age ranges. The scores of dependence (3.889 \pm 0.928$)$ of anatomists aged 36-40 are higher than those in any other age range. The scores of reward dependence (17.11 \pm 2.31$)$ of anatomists aged $36-40$ are higher than those of any others. The scores of resourcefulness (4.45 \pm 1.03$)$ of anatomists aged $41-45$ are higher than those of any others.

Differences according to the current career degrees (professor, associate professor, assistant professor, others):

The scores of extravagance $(5.73 \pm 1.71)$ of assistant professor anatomists are higher than those of others, the scores of resourcefulness of (4.47 \pm 0.73 ) professor anatomists are higher than those of others, the scores of self-acceptance $(8.05 \pm 2.20)$ of professor anatomists are higher than those of others.

Differences according to the specialty form (medical license examination (TUS), doctorate $(\mathrm{PhD})$, master degree $+\mathrm{PhD})$ :

The scores of impulsiveness $(6.50 \pm 2.50)$ of anatomists with master degree $+\mathrm{PhD}$ specialty are higher than those of others; the total scores of novelty seeking $(22.62 \pm 4.65)$ of anatomists with master degree and $\mathrm{PhD}$ specialty are higher than those of others; the scores of anticipatory worry $(5.361 \pm 2.257)$ of anatomists with doctorate specialty are higher than those of others; the scores of shyness $(3.68 \pm 2.32)$ of anatomists with TUS specialty are higher than those of others, the scores of fatigability (3.58 \pm 2.18$)$ of anatomists with doctorate specialty are higher than those of others, the total scores of harm avoidance (15.88 \pm 6.77$)$ of anatomists with doctorate specialty are higher than those of others; the scores of pure-hearted conscience $(8.500 \pm 0.756)$ of anatomists with master degree $+\mathrm{PhD}$ specialty are higher than those of others; the scores of purposefulness $(6.57 \pm 1.50)$ of anatomists with TUS specialty are higher than those of others; the scores of resourcefulness $(4.28 \pm 1.29)$ of anatomists with TUS specialty are higher than those of others; the scores of enlightened second nature (10.62 \pm 0.91$)$ of anatomists with master degree $+\mathrm{PhD}$ specialty are higher than those of others; the scores of self-forgetfulness $(6.63 \pm 2.72)$ of anatomists with doctorate specialty are higher than those of others; the scores of transpersonal identification (6.02 \pm 1.57 ) of anatomists with doctorate specialty are higher than those of others; the scores of spiritual acceptance $(9.25 \pm 1.03)$ of anatomists with master degree $+\mathrm{PhD}$ are higher than those of others.

Differences according to gender

The scores of disorderliness in women anatomists $(x=2.577)$ are lower than those in men anatomists $(x=3.793)$. The scores of fear of uncertainty in women anatomists $(x=4.692)$ are higher than those in men anatomists $(x=2.717)$. The scores of shyness in women anatomists $(x=4.308)$ are higher than those in men anatomists $(x=2.679)$. The total scores of harm avoidance in women anatomists $(x=17.039)$ are higher than those in men anatomists $(x=12.377)$. The scores of dependence in women anato-
Tab. 2. Distribution of Holland's Occupational Themes.

\begin{tabular}{lcc}
\hline & $\mathrm{n}$ & $\%$ \\
\hline Realistic & 28 & 35 \\
Investigative & 22 & 28 \\
Artistic & 6 & 8 \\
Social & 35 & 44 \\
Enterprising & 4 & 5 \\
Conventional & 15 & 19 \\
\hline Total & 125 & 100 \\
\hline
\end{tabular}

mists $(x=4.808)$ are higher than those in men anatomists $(x=3.755)$. The scores of self-acceptance in women anatomists $(x=6.346)$ are lower than those in men anatomists $(x=7.887)$. The scores of enlightened second nature in women anatomists $(x=10.346)$ are higher than those in men anatomists $(x=9.377)$.

\section{Holland's theme scores}

According to Holland test, medical school (physician) is included in investigative and social groups. Besides medical doctor, originated from veterinarian, biologist, physical therapist, and nurse are among the anatomists. Veterinarians, biologists, physical therapists, and nurses are placed in realistic and social groups, whereas the academic staff is placed in social group.

Answers to Holland test given by anatomists who joined the questionnaire are below.

Table 2 - distribution of Holland's Occupational Themes

For anatomists, the career interest was determined by Holland's Theme Codes. The order of high frequency of Holland's Codes was as follows: social $(44.3 \%)$, realistic $(35.4 \%)$, investigative $(27.8 \%)$, conventional $(19.0 \%$, artistic $(7.6 \%)$ and enterprising $(5.1 \%)$.

According to the results, anatomists are mostly placed in social group. This fact promotes the academician identity.

Results which are statistically significant

The anticipatory-worry points $(x=4.14)$ of the anatomists with realistic personality type have been found lower than in those with other personality types $(\mathrm{x}=5.03)$. The responsibility points of the anatomists with realistic personality type $(x=6.464)$ have been found higher than of those with other personality types $(x=5.62)$. The resourcefulness points $(\mathrm{x}=4.75)$ of the anatomists with realistic personality type have been found higher than in those with other personality types $(x=3.74)$; self acceptance points $(x=8.17)$ of the anatomists with realistic personality type have been found higher than in those with other personality types $(x=6.94)$; self directedness points $(x=35.42)$ in the anatomists with realistic personality type have been found higher than in those with other personality types $(x=31.90)$; the compassion points $(x=8.07)$ of the anatomists with realistic personality type have been found higher than in those with other personality types $(x=6.05)$; the cooperativeness points $(\mathrm{x}=33.00)$ of the anatomists with realistic personality type have been found higher than in those with other personality types $(x=29.37)$; the transpersonal identification points $(x=4.39)$ of the anatomists with realistic personality type have been found lower than in those with other personality types $(x=5.51)$; the spiritual acceptance points $(x=5.57)$ of the anatomists with realistic per- 
sonality type have been found lower than in those with other personality types $(\mathrm{x}=7.78)$.

The anticipatory-worry points $(x=6.00)$ of the anatomists with investigative personality type have been found higher than of those with other personality type $(x=4.22)$; the dependence points $(x=2.04)$ of the anatomists with investigative personality type have been found lower than in those with other personality type $(x=2.66)$; the persistence points $(x=4.68)$ of the anatomists with investigative personality type have been found lower than in those with other personality type $(x=5.71)$; the purposefulness points $(x=5.09)$ of the anatomists with investigative personality type have been found lower than in those with other personality type $(x=6.42)$; the resourcefulness points $(x=3.77)$ of the anatomists with investigative personality type have been found lower than in those with other personality type $(x=4.22)$; the enlightened second nature points $(x=8.63)$ of the anatomists with investigative personality type have been found lower than in those with other personality type $(x=10.10)$; the self directedness points $(x=30.40)$ of the anatomists with investigative personality type have been found lower than in those with other personality type ( $x=34.21)$; the helpfulness points $(x=4.54)$ of the anatomists with investigative personality type have been found lower than in those with other personality type $(x=5.50)$; the self-forgetfulness points $(x=7.40)$ of the anatomists with investigative personality type have been found higher than in those with other personality type $(x=5.28)$; the transpersonal identification points $(\mathrm{x}=6.00)$ of the anatomists with investigative personality type have been found higher than in those with other personality type $(x=4.77)$.

The impulsiveness points $(\mathrm{x}=2.16)$ of the anatomists with an artistic personality type have been found lower than in those with other personality type $(x=3.79)$; the disorderliness points $(x=2.33)$ of the anatomists with an artistic personality type have been found lower than in those with other personality type $(x=3.480)$; the shyness points $(\mathrm{x}=1.16)$ of the anatomists with an artistic personality type have been found lower than in those with other personality type $(\mathrm{x}=3.38)$; the fatigability points $(\mathrm{x}=0.50)$ of the anatomists with an artistic personality type have been found lower than in those with other personality type $(x=2.78)$; the harm avoidance points $(x=9.66)$ of the anatomists with an artistic personality type have been found lower than in those with other personality type $(\mathrm{x}=14.26)$; the dependence points $(\mathrm{x}=3.33)$ of the anatomists with an artistic personality type have been found higher than in those with other personality type $(x=2.42)$; the responsibility points $(x=7.16)$ of the anatomists with an artistic personality type have been found higher than in those with other personality type $(x=5.82)$; the empathy points $(x=6.00)$ of the anatomists with an artistic personality type have been found higher than in those with other personality type $(\mathrm{x}=4.78)$.

The impulsiveness points $(\mathrm{x}=4.05)$ of the anatomists with a social personality type have been found higher than in those with other personality type $(x=3.36)$; the anticipatory worry points $(x=5.14)$ of the anatomists with a social personality type have been found higher than in those with other personality type $(x=4.38)$; the fatigability points $(x=3.20)$ of the anatomists with a social personality type have been found higher than in those with other personality type $(x=2.13)$; the responsibility points $(x=5.45)$ of the anatomists with a social personality type have been found lower than in those with other personality type $(x=6.29)$; the purposefulness points $(\mathrm{x}=5.45)$ of the anatomists with a social personality type have been found lower than in those with other personality type $(x=6.52)$; the resourcefulness points $(x=3.74)$ of the anatomists with a social personality type have been found lower than in those with other personality type ( $\mathrm{x}=4.38)$; enlightened second nature points $(x=9.14)$ of the anatomists with a social personality type have been found lower than in those with other personality type $(x=10.13)$; the self directedness points $(x=30.82)$ of the anatomists with a social personality type have been found lower than in those with other personality type $(x=35.00)$; the self forgetful points $(x=6.68)$ of the anatomists with a social personality type have been found higher than in those with other personality type $(x=5.22)$; the transpersonal identification points $(x=5.71)$ of the anatomists with a social personality type have been found higher than in those with other personality type $(x=4.63)$.

The exploratory excitability points $(x=8.75)$ of anatomists with an enterprising personality type have been found higher than in those with other personality type $(x=6.13)$; the enlightened second nature points $(\mathrm{x}=12.00)$ of anatomists with an enterprising personality type have been found higher than in those with other personality type $(x=9.57)$; the social acceptance points $(x=4.00)$ of the anatomists with a social personality type have been found lower than in those with other personality type $(x=6.45)$; the helpfulness points $(\mathrm{x}=4.00)$ of the anatomists with a social personality type have been found lower than in those with other personality type $(x=5.30)$; the compassion points $(x=2.75)$ of the anatomists with a social personality type have been found lower than in those with other personality type $(x=6.98)$; the pure hearted conscience points $(x=5.75)$ of the anatomists with a social personality type have been found lower than in those with other personality type $(x=7.53)$; the cooperativeness points $(x=21.00)$ of the anatomists with a social personality type have been found lower than in those with other personality type $(x=31.17)$.

The disorderliness points $(\mathrm{x}=2.13)$ of anatomists with a conventional personality type have been found lower than in those with other personality type $(x=3.68)$; the novelty seeking points $(x=14.33)$ of anatomists with a conventional personality type have been found lower than in those with other personality type $(x=18.59)$; the anticipatory worry points $(x=5.46)$ of anatomists with a conventional personality type have been found higher than in those with other personality type $(x=4.54)$; the fear of uncertainty points $(x=5.80)$ of anatomists with a conventional personality type have been found higher than in those with other personality type $(x=2.79)$; the shyness points $(x=6.26)$ of anatomists with a conventional personality type have been found higher than in those with other personality type $(x=2.500)$; the fatigability points $(\mathrm{x}=4.20)$ of anatomists with a conventional personality type have been found higher than in those with other personality type $(\mathrm{x}=2.23)$; the harm avoidance points $(\mathrm{x}=12.07)$ of anatomists with a conventional personality type have been found lower than in those with other personality type $(x=21.73)$; the persistence points $(x=3.46)$ of anatomists with a conventional personality 


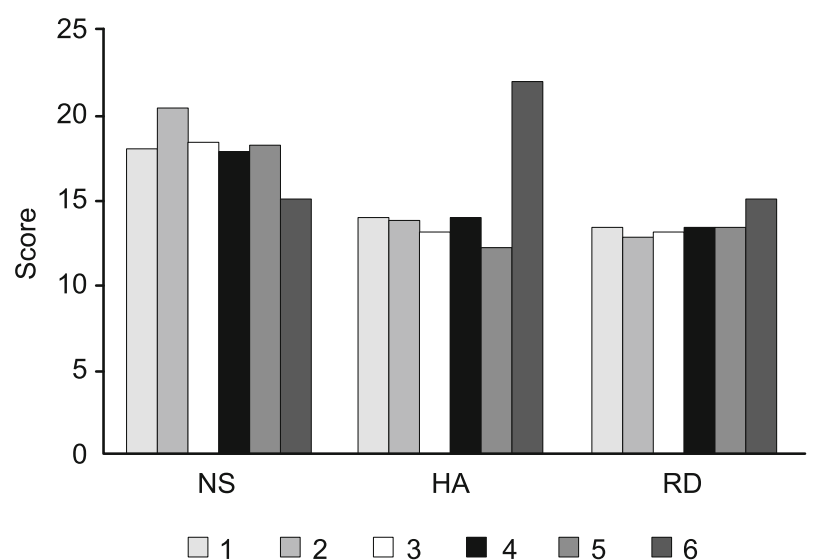

Fig. 1. Relationship between reasons why anatomists have chosen anatomy and their personal profiles were examined.

type have been found lower than in those with other personality type $(x=5.89)$; the resourcefulness points $(x=3.60)$ of anatomists with a conventional personality type have been found lower than in those with other personality type $(x=4.21)$; the self acceptance points $(x=5.86)$ of anatomists with a conventional personality type have been found lower than in those with other personality type $(x=7.73)$; the enlightened second nature points $(x=11.00)$ of anatomists with a conventional personality type have been found higher than in those with other personality type ( $x=9.39)$; the helpfulness points $(x=4.33)$ of anatomists with a conventional personality type have been found lower than in those with other personality type $(\mathrm{x}=5.45)$.

\section{Reasons for choosing anatomy}

Twenty-two options were given as the reasons for choosing anatomy (Supp 1). The most frequently chosen ones are as follows:

According to the questionnaire, the most important cause in choosing anatomy is the interest in anatomy since medical study time. Second important cause is 'I would use it as a step'.

Relationship between reasons why anatomists have chosen anatomy and their personal profiles were examined (Fig. 1). For this, the components of the personal test, namely NS (novelty seeking), HA (harm avoidance) and RD (reward dependence) were compared to reasons for anatomists to choose anatomy (10). Aggregating similar options, reasons for anatomists to choose anatomy were reduced to six options (Tab. 3).

Table 3 - reasons for choosing anatomy
Figure 1 - relationship between reasons of anatomists' choice and personality profiles

Statistically significant results are as follows.

Extravagance scores of anatomists who selected second choice ( $\mathrm{PhD}$ degree without taking medical license examination) is higher than those in people entering for TUS examinations $(\mathrm{p}=0.033)$, and novelty seeking scores are found to be higher than in those not selecting the second choice $(\mathrm{p}=0.045)$. Fatigability scores of the anatomists who selected the third choice (not willing to see or examine the patients) were found lower than in those not selecting the third choice $(p=0,009)$.

According to these results, the personality profiles of anatomists have not affected the motivation to select their specialty choice (career) except for the above results (Fig. 1) which had a partial effect.

\section{Discussion}

Many physicians have studied the relationship between a doctor's personality and chosen specialty. A group of surgeons sought to determine whether there were differences in the characteristics and temperament of physicians in three types of medical careers, namely surgery, primary care (family practice, internal medicine, and pediatrics), and controllable lifestyle specialties (anesthesiology, dermatology, emergency medicine, neurology, ophthalmology, pathology, psychiatry, and radiology). Most students think of surgeons as dominant, uninhibited, and aggressive. They tend to overlook the fact that surgery requires a certain type of person who can handle its tasks and challenges (4).

Another landmark study surveyed a group of medical students to determine any relationships between personality type and specialty choice. Students entering the hospital-based specialties (anesthesiology, radiology, or emergency medicine) had less tolerance for ambiguity and preferred highly structured environments with fixed guidelines. Future obstetricians and gynecologists saw themselves as warm and helpful, but they were also emotionally vulnerable, uncomfortable around others, and very concerned about appearances and making a good impression. Future pediatricians, who sought warm and close interactions with their patients, were the most extroverted and sociable people. In contrast, the introverted students with fewer social connections, particularly the ones who had been in psychotherapy themselves, became psychiatrists. The study also found that students interested in surgery were more likely to be competitive, aggressive, and highly confident. They were the doctors-to-be who carried a strong conviction that their actions could rapidly influence the course of events (4).

Tab. 3. Reasons for choosing anatomy.

\begin{tabular}{|c|c|c|c|c|c|}
\hline \multicolumn{6}{|l|}{ Descriptive Statistics } \\
\hline & $\mathrm{n}$ & Minimum & Maximum & Mean & $\mathrm{SD}$ \\
\hline Interested in since medical student (1) & 79 & 1 & 99 & 45.66 & 49.119 \\
\hline Having PhD degree without taking medical license exam (2) & 79 & 1 & 99 & 85.35 & 34.144 \\
\hline Not willing to see or examine the patients (3) & 79 & 1 & 99 & 41.94 & 48.641 \\
\hline Activities required for the occupation (4) & 79 & 1 & 99 & 9.68 & 28.027 \\
\hline Use anatomy as a step (5) & 79 & 1 & 99 & 77.91 & 40.531 \\
\hline Enjoying philosophy, interested in life and death (6) & 1 & 1 & 1 & 1.00 & . \\
\hline
\end{tabular}


$112-118$

Anesthesiologists were self-confident, had superior interpersonal skills, and were goal seeking, as evidenced by high scores on CPI scales of dominance, social presence, and achievement via independence, respectively. Family practitioners were predominately sensors $(\mathrm{S})$, thinkers $(\mathrm{T})$, and perceivers $(\mathrm{P})$. Obstetricians and Gynecologists were higher on sensing-thinking-judging (STJ). Pediatricians showed a large proportion of extroversion-sensingfeeling-judging (ESFJ) and introverted-sensing-feeling-judging (ISFJ). Intuition (N) was followed by thinking, sensing, and feeling (TSF) for physiatrists.

Psychiatrists have greater frustration tolerance, emotional maturity, stability, and reality orientation. Surgeons were found to be more flexible than nonsurgical physicians. Surgeons are extroverted (E) sociable and active. Pathologists tended to display introversive (I), intuitive (N), and thinking (T) dimensions. Internists have a tendency to be stiff, cool, skeptical, and aloof and they prefer working with things rather than people (1).

No study was seen in literature regarding the personality analysis of anatomists. Only the rate of selection of anatomy and view on anatomy was investigated among medical students of Nigeria. Although most students agreed that anatomy was an important subject in medical sciences, namely 346 (98\%), only $22(6.2 \%)$ would choose anatomy as a career. Male students were more likely to choose anatomy as a career (9).

According to our study, special personality traits of the anatomists' are as follows:

Temperament components of the TCI were novelty seeking (mean $=17.7 \pm 4.7)$, harm avoidance $($ mean $=13.9 \pm 6.1)$, reward dependence $($ mean $=13.2 \pm 3.4)$, and persistence $($ mean $=5.4 \pm 2.1)$. Character profiles included self-directedness $($ mean $=33.1 \pm 6.3)$, cooperativeness $($ mean $=30.6 \pm 5.9)$, and self-transcendence (mean $=17.9 \pm 5.6$ ).

The order of high frequency Holland's Codes was social $(44.3 \%)$, realistic $(35.4 \%)$, investigative $(27.8 \%)$, conventional (19.0\%), artistic (7.6\%), and enterprising (5.1\%).

\section{Reasons for choosing anatomy}

According to the last questionnaire, the most important cause for choosing anatomy is the interest in anatomy since medical study time. Second important cause is 'I would use it as a step'.

According to our study, personality specialty of anatomists has affected the motivation in career selection in part.

According to Hollands code, anatomists mostly describe themselves as social which means that they like to work with people to enlighten, help, train or cure them, or are skilled with words. According to their second choice, they are realistic, prone to be honest, humble, natural, persistent, practical, shy, and thrifty. Anatomists can be described as curious, independent, modest, precise, rational, reserved, and smart as well since their third choice describes them as investigative persons. These results in part support John Holland's theory (8), which takes the career as a function of personality and has been one of the most widely accepted theories in this area.

Jobs consist of two components (1). For physicians, the first component deals with using biotechnical competencies to perform specific tasks that distinguish their specialty. The second compo- nent deals with the context of task performance and involves maintenance of the social and organizational network that surrounds the tasks. This suggests the possibility that personality traits may relate differentially to the two components of physicians' work. The personality may relate more to contextual performance than task performance. This means that, despite doing similar tasks, physicians in a particular specialty may exhibit variations in contextual performance that reflect a wide range of personality traits. This would explain why personality measures would not be particularly effective in predicting medical specialty choice. It also suggests that future research on medical specialty selection should concentrate on task performance by measuring skills, self-efficacy, attitudes, and aptitudes rather than personality.

Medical specialty choice might be a common instrument in diverse studies of the personality and the decision-making process medical students use to choose their specialties (1).

Medical career selection is a complex process. Our study revealed that besides specialty characters, personality preferences are also important factor for the selection.

Inspection of complex relationship among TCI profile, age, gender, and motivation to select the career can be used when considering the choice for anatomy department or inducing anatomy workers to manage their job.

\section{References}

1. Borges NJ, Savickas ML. Personality and Medical Specialty Choice: A Literature Review and Integration. J Career Assess 2002; 10 (3): 362-380.

2. Schwartz RW, Barclay JR, Harrell PL, Murphy AE, Jarecky RK, Donnelly MB. Defining the surgical personality: A preliminary study. Surgery 1994; 115: 62-68.

3. Borman WC, Motowidlo SJ. Expanding the criterion domain to include elements of contextual performance. In: Schmitt N, Borman WC (Eds). Personnel selection in organizations. 71-98. San Francisco: Jossey-Bass, 1993.

4. Freeman B. Choosing a medical speciality. New York: McGraw-Hill Medical, 2004.

5. Reeve PE. Personality characteristics of a sample of anaesthetists. Anaesthesia 1980; 35: 559-568.

6. Cloninger CR, Svrakic DM, Przybeck TR. A psychobiological model of temperament and character. Arch Gen Psych 1993; 5: 975-990.

7. Köse S, Sayar K, Kalelioglu U et al. Turkish version of the TCI: Reliability, validity, and factorial structure. Bull Clin Psychopharmacol 2004; 14: 107-131.

8. Holland JL. Making vocational choices: a theory of careers. Englewood Cliffs: Prentice-Hall, 1973.

9. Onakpoya OH, Onakpoya UU, Adereti GE. The prospect of anatomy as a career choice among clinical year medical students in Nigeria. Ann Afr Med 2009; 8 (2): 90-94.

10. Vaidya NA, Sierles FS, Raida MD, Fakhoury FJ, Przybeck TR, Cloninger CR. Relationship between specialty choice and medical student temperament and character assessed with Cloninger Inventory. Teach Learn Med 2004; 16 (2): 150-156.

Received April 22, 2015. Acceptance September 20, 2015. 